\title{
ESCALA DE LOCUS DE CONTROLE DA SAÚDE - MHLC: ESTUDOS DE VALIDAÇÃO
}

\author{
Jesús Edilberto Rodríguez-Rosero ${ }^{1}$ \\ Maria das Graças Carvalho Ferriani ${ }^{2}$ \\ Marilia Ferreira Dela Coleta ${ }^{3}$
}

Rodríguez-Rosero JE, Ferriani MGC, Dela Coleta MF. Escala de locus de controle da saúde - MHLC: estudos de validação. Rev Latino-am Enfermagem 2002 março-abril; 10(2):179-84.

Este estudo trata dos procedimentos para adaptação e validação da escala de locus de controle da saúde - MHLC, traduzida para o Espanhol. A coleta de dados foi realizada em Villavicencio -Colômbia, e contou com a participação de 242 mulheres, com idade variando de 22 a 33 anos. Diversos tratamentos estatísticos foram utilizados para verificar as qualidades psicométricas desse instrumento. Foram observadas, também, diferenças nos escores, em função da escolaridade de subgrupos, de modo consistente com a literatura. Os resultados demonstram que as escalas possuem confiabilidade e validade suficiente para ser usadas na língua espanhola, no contexto Colombiano.

DESCRITORES: saúde, enfermagem, mães

\section{MULTIDIMENSIONAL HEALTH LOCUS OF CONTROL SCALE - MHLC: A VALIDATION STUDY}

The study aimed at validating a multidimensional health locus of control scale - MHLC and was developed with the participation of 242 women from the city of Villavicencio - Colombia. Several statistics treatments were used with the purpose to confirm the psychometric qualities of this instrument. The authors found differences among the scores, due to the educational level of the subgroups, according to the literature. Results showed the reliability and validity of the scales written in Spanish and adapted to Colombian context.

DESCRIPTORS: health, nursing, mothers

\section{ESCALA DE LOCUS DE CONTROL DE LA SALUD - MHLC: ESTUDIOS DE VALIDACIÓN}

Este estudio trata de los procedimientos para adaptación y validación de la escala de locus de control de la salud - MHLC, traducida para el Español. Los datos fueron recolectados en Villavicencio - Colombia y contó con la participación de 242 mujeres, cuya edad osciló entre 22 a 33 años. Diversos tratamientos estadísticos fueron utilizados para verificar las cualidades psicométricas de este instrumento. Fueron observadas diferencias en los escores en función de la escolaridad de subgrupos de modo consistente con la literatura. Los resultados demuestran que las escalas poseen confiabilidad y validez suficiente para ser usadas en la lengua española en el contexto Colombiano.

DESCRIPTORES: salud, enfermería, madres

\footnotetext{
${ }^{1}$ Professor Titular da Universidad de Los Llanos Colombia, e-mail: heidyr@impsat.net.co; ${ }^{2}$ Professor Titular da Escola de Enfermagem de Ribeirão Preto da Universidade de São Paulo, Centro Colaborador da OMS para o desenvolvimento da pesquisa em enfermagem, e-mail: caroline@eerp.usp.br; ${ }^{3}$ Professor Titular da Universidade Federal de Uberlânddia, e-mail: dcoleta@Inx.triang.com.br
} 
INTRODUÇÃO

A interdisciplinaridade mostra-se cada vez mais importante nos estudos e na atuação em saúde. Diversas variáveis psicológicas têm sido investigadas quanto ao seu papel na promoção da saúde, sendo grande o número de pesquisas, a partir dos anos 70 , sobre a variável locus de controle.

Locus de controle é um construto criado para explicar a percepção das pessoas sobre quem ou o que detém o controle sobre sua vida. Essa tendência manifesta-se nas expectativas individuais de alcançar resultados desejados no futuro e está relacionada ao comportamento na medida em que esses resultados são percebidos como relevantes para o sujeito e como prováveis de ocorrer ${ }^{(1)}$.

O mesmo autor referiu-se ao Locus de controle como uma expectativa generalizada, uma tendência em perceber os eventos da vida como controlados pelo próprio sujeito. Nesse caso, o Locus seria interno, ou, por outro lado, como controlado por outros fatores, que não ele próprio, ou Locus externo. Os fatores externos poderiam ser outras pessoas, entidades, o destino, a sorte ou as situações. Esse autor também construiu um instrumento para medida do locus de controle. Sua escala fornece um escore que permite identificar a tendência "interna" ou "externa" na percepção de controle que as pessoas apresentam ${ }^{(2)}$. Colocado dessa forma, o construto seria unidimensional, e seus pólos extremos seriam a internalidade e a externalidade.

Diversos pesquisadores estudaram a dimensionalidade da escala, mais intensamente durante os anos 70 , encontrando uma diversidade de fatores ${ }^{(3)}$. Buscou-se construir sua escala considerando o construto com três dimensões, uma interna e duas externas. As duas dimensões externas mediriam a percepção de controle por pessoas poderosas (externos-outros poderosos) e pelo acaso, azar, sorte, ou destino (externos-acaso ou externos autênticos). Portanto, é possível verificar-se o grau em que as pessoas acreditam estar no controle de suas vidas, o quanto elas pensam que esse controle está nas mãos de pessoas poderosas, e o grau em que elas acreditam que a vida é controlada pelo acaso ou destino.

O Locus de controle, inicialmente como medida generalizada, gerou estudos e medidas específicas para aspectos particulares da vida do indivíduo, tais como o trabalho, a escola, 0 casamento ou a saúde, sendo aceito, atualmente, que é possível, ao mesmo tempo, ser interno a respeito de um tema, e externo ao se considerar outro.

Os primeiros estudos na área especifica da saúde, para verificar a relação do Locus de controle com diversos comportamentos relacionados à prevenção, tratamento e controle de variadas doenças e disfunções, foram fundamentados em escalas específicas para medida do Locus de controle da saúde, que foram posteriormente desenvolvidos, uma unidimensional e outra multidimensional, seguindo-se diversos instrumentos ainda mais específicos, relativos a alcoolismo, saúde mental, diabetes, obesidade, artrite, depressão, câncer, hipertensão, fumo, dor, doença cardíaca, incapacidade física, saúde bucal e disfunções sexuais ${ }^{(1,3-6)}$. O desenvolvimento da escala multidimensional de Locus de controle da saúde permitiu verificar que dimensões de controle mais se relacionavam aos problemas específicos de saúde, se interno (percepção de que o sujeito controla e é responsável pela própria saúde), externo- outros poderosos (percepção de que os profissionais de saúde ou a família detêm 0 controle da saúde do indivíduo) ou externo-acaso (percepção de que a saúde depende do acaso, sorte ou destino).

A mais extensa das revisões sobre Locus de controle e saúde apresentada, onde os sujeitos internos mostram sempre melhores resultados e comportamentos de saúde mais adequados relativos a: tabagismo, vacinação, exercícios, obesidade, controle da natalidade, coronariopatias, entre outros. Em geral, as amostras de doentes crônicos ou graves eram mais externas que pacientes menos graves ou que grupos de controle. Indivíduos internos também procuram, mais do que os externos, informações sobre sua doença ${ }^{(7)}$.

A maioria dos estudos utiliza as escalas de Locus de controle da saúde ${ }^{(8-9)}$. Os resultados são semelhantes e referem-se ao melhor desempenho dos sujeitos internos em deixar de fumar, perder peso, controlar o diabetes, controlar a pressão arterial, tomar medicações, conhecer sua doença, usar aparelhos corretivos, reabilitar-se, colaborar na diálise e na fisioterapia ${ }^{(7)}$.

No Brasil, alguns estudos realizados com a escala multidimensional de Locus, de controle da saúde (MHLC), apresentam resultados consistentes com aqueles obtidos em outras culturas ${ }^{10-}$ 12).

Este estudo tem o objetivo de testar as qualidades psicométricas da escala MHLC, traduzida para o espanhol, em uma amostra de sujeitos colombianos.

\section{MÉTODO}

Sujeitos

Participaram 242 mulheres, sendo 76 profissionais de Enfermagem -grupo (E) e 166 mães -grupo (M). Na amostra (M), aplicaram-se 200 testes, dos quais foram eliminados 44 sujeitos por não terem preenchido todos os itens, tendo como resultado: $(M)=166$ sujeitos. No grupo $(\mathrm{E})$, foram aplicados 100 testes, dos quais foram eliminados 24 sujeitos, por não terem preenchido todos os itens, tendo como resultado: $(E)=76$ sujeitos.

A amostra (M) tem uma média de idade de 27,9 anos, todas 
possuem pelo menos um filho menor de cinco anos, e $96 \%$ têm entre 1 e 3 filhos. A amostra (E) tem uma média de idade de 24,7 anos, não possuem filhos e apresentam nível superior de escolaridade. É necessário esclarecer que, para se formar um profissional de nível superior (enfermeiro) na Colômbia, é necessário ter cursado, com sucesso, 5 anos de escola primária, 6 anos de nível secundário e 4 anos de nível superior.

Instrumento

A escala MHLC foi construída a partir dos 11 itens da escala $\mathrm{HLC}^{(4)}$, incluindo novos itens, no sentido de medir as três dimensões pretendidas: internalidade (IHLC), externalidade outros poderosos (PHLC) e externalidade acaso (CHLC), seguindo proposta multidimensional $^{(3)}$.

Cada uma das três sub-escalas apresenta seis itens referentes às dimensões:

Internalidade para a saúde (Internality Health Locus of controle), no sucessivo se denominará (I).

Externalidade; Outros poderosos para a saúde (Powerful other Health Locus of control), no sucessivo se denominará (P). Externalidade; Acaso para a saúde (Chance Health Locus of control), no sucessivo se denominará $(C)$.

Os escores fornecem, respectivamente, o grau em que 0 sujeito acredita em si mesmo, em pessoas poderosas, e 0 acaso como fonte de controle de sua saúde. Os 18 itens são apresentados aos sujeitos como uma escala única e respondidos na forma Likert, com cinco níveis de resposta, conforme se segue: $1=$ Concorda totalmente. 2= De acordo em sua maior parte. $3=$ Em dúvida. 4= Em desacordo em sua maior parte. $5=$ Totalmente em desacordo.

Para obter os escores, somam-se os números dos itens correspondentes: Sub-Escala I: Somar os itens 1, 6, 8, 12, 13, 17. Sub-Escala P: Somar os itens 3, 5, 7, 10, 14, 18. Sub-Escala C: Somar os itens $2,4,9,11,15,16$. Os escores variam entre 6 e 30 pontos; quanto maior o valor, menor a crença de que cada fator respectivo controle a sua saúde.

Procedimentos

Processo da procura das amostras

Para a adaptação da escala Locus, de controle da saúde, para amostras Colombianas, utilizou-se a versão em português ${ }^{(12)}$, que foi traduzida para o espanhol (Anexo 1).

Em seguida foram selecionadas as duas amostras na cidade de Villavicencio-Colômbia, durante os meses de fevereiro a maio de 1997. A amostra específica de mães (M) foi obtida em onze escolas públicas de primeiro grau e duas creches também públicas que foram denominadas instituições-janela, todas localizadas em zona urbana.

Questionários exploratórios para obter a amostra foram encaminhados com alunos do $1^{\circ} \mathrm{e} 2^{\circ}$ anos; as crianças levaram para suas famílias 3.089 questionários do tipo exploratório, com o conteúdo mínimo, apresentado a seguir.

() DADOS DA MÄ́E. NOME: ENDEREÇO: GRAU DE ESTUDO: IDADE: INICIANDOPELOMENOR, ESCREVAAIDADE DOS FILHOS VIVOS:

TRABALHANO SETOR SAÚDE? SIM NOME DOALUNO: NÁO CURSO:

Esse questionário teve como finalidade a obtenção das informações necessárias para encontrar o grupo-critério, resultando $2.093(67,76 \%)$ retornos. Analisadas essas informações, foram encontradas 506 (16,38\%) mães que se enquadravam nos critérios de seleção; a essas mães foi encaminhado um convite pessoal, obtendo-se a colaboração de 200 (6,47\%) sujeitos, constituindo, assim, as mães-alvo da aplicação dos 3 instrumentos em grupos que variaram de 5 a 17 mães por vez.

As enfermeiras $(E)$ foram abordadas em locais de trabalho e em seus domicílios, também solicitando-lhes a colaboração com o estudo.

A sistematização dos dados foi realizada no Brasil. Criouse um banco de dados no programa "EXCEL 6.0", para execução dos cálculos e, como fonte matriz de alimentação para as provas estatísticas especiais realizadas, foi utilizado o software "Statistical Package for the Social Science" (SPSSWIN). Os testes estatísticos foram utilizados com o objetivo de comprovar a qualidade da escala para aplicação em amostras de língua espanhola.

\section{RESULTADOS}

Confiabilidade

O alfa de Cronbach é uma técnica utilizada quando a resposta ao item pode assumir mais de duas alternativas. Seu resultado é um indicador sumário da consistência interna da escala analisada e, conseqüentemente, dos itens que a compõem. A vantagem da utilização desta técnica é a possibilidade de se obter uma medida tão confiável e tão válida quanto possível, utilizando o menor número de itens, isto é, selecionar itens que contribuam ao máximo para a confiabilidade e validade. 
Tabela 1 - Estatísticas para I, P, C nas amostras (E) e (M)

\begin{tabular}{lcccc}
\hline Instrumento & $\bar{X}$ & Md & Mo & $s$ \\
\hline I(E) & 13,5 & 14 & 15 & 3,6 \\
I(M) & 12,9 & 13 & 15 & 3,8 \\
P(E) & 19,2 & 19 & 19 & 4,1 \\
P (Mi) & 13,1 & 12 & 12 & 5,0 \\
C (E) & 24,9 & 26 & 28 & 3,6 \\
C(M) & 19,5 & 20 & 22 & 5,1 \\
\hline
\end{tabular}

Tabela 2 - Valores de p e $t$ calculados tomando o $25 \%$ superior dos escores $n=242$

\begin{tabular}{ccc}
\hline Hens & Valor $p$ & Teste $\mathrm{t}$ \\
\hline 1 & $3,45 \mathrm{E}-18$ & 12,57 \\
2 & $2,89 \mathrm{E}-53$ & 54,99 \\
3 & $1,77 \mathrm{E}-41$ & 35,33 \\
4 & $4,80 \mathrm{E}-71$ & 40,15 \\
5 & $7,93 \mathrm{E}-52$ & 54,02 \\
6 & $1,00 \mathrm{E}-34$ & 26,97 \\
7 & $1,55 \mathrm{E}-59$ & 73,55 \\
8 & $8,49 \mathrm{E}-54$ & 56,15 \\
9 & $1,71 \mathrm{E}-56$ & 62,38 \\
10 & $4,70 \mathrm{E}-81$ & 181,09 \\
11 & $1,09 \mathrm{E}-35$ & 27,24 \\
12 & $1,70 \mathrm{E}-39$ & 32,95 \\
13 & $4,97 \mathrm{E}-19$ & 13,15 \\
14 & $1,51 \mathrm{E}-32$ & 24,63 \\
15 & $1,57 \mathrm{E}-52$ & 53,44 \\
16 & $2,09 \mathrm{E}-34$ & 25,02 \\
17 & $6,64 \mathrm{E}-36$ & 28,38 \\
18 & $1,45 \mathrm{E}-67$ & 100,65 \\
\hline
\end{tabular}

$\mathrm{I}=$ Internos, itens $1,6,8,12,13,17$

$\mathrm{P}=$ Outros poderosos, itens $2,4,9,11,15,16$

$C=$ Destino, acaso, itens $3,5,7,10,14,18$

Tabela 3 - Coeficientes alfa de Cronbach das escalas de Locus de controle da saúde, em sua versão original em inglês (Wallston e Wallston, 1981)

\begin{tabular}{lcccc}
\hline Amostra & $\begin{array}{l}\mathrm{N} \\
\propto\end{array}$ & $\begin{array}{l}\mathrm{HLC} \\
\propto\end{array}$ & $\begin{array}{l}\mathrm{PHLC} \\
\propto\end{array}$ & $\begin{array}{l}\mathrm{CHLC} \\
\propto\end{array}$ \\
\hline Universitánios & 86 & 0.66 & 0.64 & 0.47 \\
Universitános & 85 & 0.72 & 0.56 & 0.70 \\
Universitários & 112 & 0.61 & 0.56 & 0.55 \\
Adultos & 82 & 0.66 & 0.56 & 0.65 \\
\hline
\end{tabular}

De acordo com os resultados demonstrados na Tabela 3, no presente estudo, as escalas de externalidade (alfa $=0,71$ e 0,72) apresentaram melhor consistência interna do que a escala de internalidade (alfa $=0,53$ ). Conforme as Tabelas 1 e 2, os valores dos índices Alpha de Cronbach para estas três sub-escalas I, P e C, apresentados na literatura científica para instrumentos de pesquisa, são iguais ou maiores que 0,51 , portanto semelhantes aos valores encontrados no presente estudo. Considerando-se o número pequeno de itens, esses índices apresentam suficiente magnitude.
Estrutura fatorial

Conforme já exposto, a escala MHLC contém 18 itens e, em seu interior, discrimina 3 tipos de sub-agrupamentos: internalidade (I), outros poderosos $(\mathrm{P})$ e acaso (C); sendo que os dois últimos medem a crença na externalidade para a saúde.

Para confirmar essa estrutura na tradução da MHLC para a língua espanhola, foi feita uma análise dos componentes principais, encontrado-se três componentes com autovalores maiores do que 1, que representam $42,8 \%$ da variância total.

Para dicriminar melhor, executamos extração com três fatores e 5 interações cujos resultados são apresentados na Tabela 4.

Tabela 4 - Coeficientes alfa de Cronbach das escalas de Locus de controle da saúde, em sua versão traduzida para o português (Dela Coleta, 1995)

\begin{tabular}{lllll}
\hline \multicolumn{1}{c}{ Amostra } & $\begin{array}{l}\mathrm{N} \\
\propto\end{array}$ & $\begin{array}{l}\text { HLC } \\
\propto\end{array}$ & $\begin{array}{l}\text { PHLC } \\
\propto\end{array}$ & $\begin{array}{l}\text { CHLC } \\
\propto\end{array}$ \\
\hline Mulheres & 315 & 0.63 & 0.63 & 0.78 \\
Universitánios & 580 & 0.62 & 0.63 & 0.59 \\
Adultos jovens & 149 & 0.67 & 0.62 & 0.51 \\
Adultos 40 a 70 anos & 232 & 0.63 & 0.71 & 0.71 \\
\hline
\end{tabular}

Essa análise demonstrou que o fator 1 foi constituído pelos itens da escala P, menos um item (MHLC7); o fator 2 foi conformado por todos os itens da escala $\mathrm{C}$ e o fator 3 agrupou os itens da escala I, com exceção do item (MHLC1). A Tabela 4 mostra as cargas fatoriais de cada item nos três fatores, podendo-se observar valores maiores do que 0,30 e mais altos nos fatores a que teoricamente pertencem.

A rotação com 3 fatores mostrou 0 agrupamento quase perfeito das 3 dimensões originais da escala MHLC.

Diferenças entre os grupos

Tendo sido demonstrada a adequação da escala MHLC, traduzida para o espanhol, tratou-se de verificar possiveis diferenças nos escores dos dois grupos (E) e (M). Para comparação das médias dos escores obtidos por cada grupo, em cada escala, o teste estatístico utilizado foi o $t$ de Student.

Tabela 5 - Coeficientes alfa de Cronbach das escalas de Locus de controle da saúde, em sua versão traduzida para o espanhol (Rodríguez-Rosero, 1998)

\begin{tabular}{cclll}
\hline Amostra & $\begin{array}{c}\mathrm{N} \\
\propto\end{array}$ & $\begin{array}{l}\mathrm{HLC} \\
\propto\end{array}$ & $\begin{array}{l}\mathrm{PHLC} \\
\propto\end{array}$ & $\begin{array}{l}\mathrm{CHLC} \\
\propto\end{array}$ \\
\hline Mulheres 20 a 34 anos & 242 & 0.53 & 0.71 & 0.72 \\
\hline
\end{tabular}


Os grupos $(E)$ e $(M)$ diferem significativamente nas três escalas e, de modo mais marcante, nas escalas de externalidade. As diferenças indicam que o grupo $(E)$, de nível mais alto de escolaridade e representando profissionais de saúde, apresenta escores indicando muito menos externalidade para a saúde, do que o grupo (M), tanto na escala Outros Poderosos ( $t=9,29$ e $p<0,0001$ ) quanto na escala Acaso ( $t=8,33 ; p<0,0001)$. Na escala de Internalidade para a saúde, o grupo de mães, apresentou crença um pouco maior $(t=1,16 ; p<0,02)$ do que o grupo das enfermeiras.

É importante destacar as diferenças entre os grupos (E) e (M), porque confirmam estudos em diversas culturas onde se verificou que as pessoas de nível mais baixo de escolaridade são mais externas para a saúde. Também era de se esperar que enfermeiros devessem acreditar, mais que a amostra de mães, que a saúde não é devida ao acaso. Na escala de Internalidade o resultado não foi consistente com a literatura, dado que esperava-se que as enfermeiras acreditassem ter mais controle sobre a saúde do que as mães acreditavam.

\section{CONCLUSÃO}

O estudo das características psicométricas das escalas de locus, de controle da saúde, aplicadas numa amostra colombiana, em sua versão em espanhol, mostraram parâmetros de validade e fidegnidade significativos os quais indicam que estas escalas podem ser utilizadas neste pais e em outros de língua espanhola, tal como no Brasil. Todos os seus itens possuem poder de discriminação, os

\section{ANEXO 1}

\section{ESCALA MULTIDIMENSIONAL PARA LOCUS DE CONTROL EN SALUD (MHLC)}

Instrucción: Las siguientes frases expresan lo que las personas piensan y/ o hacen en asuntos de salud. Colocando un círculo en uno de los números que están al frente de cada afirmación, dé su opinión, de acuerdo con los siguientes criterios:

$1=$ Totalmente de acuerdo

$2=$ De acuerdo en su mayor pate

$3=$ En duda

$4=$ En desacuerdo en su mayor parte

$\mathbf{5}=$ Totalmente en desacuerdo

No existen respuestas correctas o erradas; lo que importa es su opinión. Por favor conteste todos.

1- Si yo estuviera enfermo, la recuperación rápida va a $\quad 1-2-3-4-5$ depender de mi comportamiento.

2- No importa lo que yo haga; si fuera para estar enfermo, $\quad 1-2-3-4-5$ enfermo de todas maneras.

3- Para mi, la mejor manera de evitar enfermedades es $\quad 1-2-3-4-5$ hacer consultas regulares con un médico.

4- Muchas cosas que afectan mi salud ocurren por $1-2-3-4-5$ casualidad. índices de confiabilidade são adequados, bem como a estrutura fatorial. Além dos resultados referentes à escala MHLC, os grupos que constituíam a amostra total foram comparados, resultando em diferenças ATRIBUIDAS ÀS CARACTERÍSITICAS RELATIVAS À ESCOLARIDADE EÀ PROFISSÃO. As maiores e mais significativas diferenças entre as duas amostras foram encontradas nas escalas de externalidade, sendo que as mães acreditam mais que pessoas poderosas para a saúde (médico, pessoal de saúde, família), bem como o acaso (destino ou azar) controlam mais a sua saúde do que as enfermeiras acreditam.

Diferentes estudos conduzidos em todo o mundo confirmam que maior internalidade do Locus de controle tende a estar associada ao sexo masculino, em nível de escolaridade mais alto, a elevados níveis socioeconomicoculturais ${ }^{(13)}$, o que tem se repetido nos estudos brasileiros $^{(14-16)}$, seja utilizando a escala unidimensional ou a multidimensional de Locus de controle generalizado.

As mesmas diferenças entre grupos etários, de escolaridade e de renda, foram verificadas também em estudos brasileiros que utilizaram medidas do locus de controle específico da saúde ${ }^{(11-12)}$.

Neste estudo com dois grupos de mulheres colombianas, com níveis de escolaridade diferentes, as maiores diferenças foram encontradas nas escalas de externalidade, de modo consistente com os estudos desenvolvidos em outras culturas e também no Brasil. $\mathrm{Na}$ escala de Internalidade, foi verificada uma pequena diferença entre as amostras, no sentido inverso ao teoricamente esperado.

Os resultados sugerem que a escala MHLC, em sua versão em espanhol, pode ser utilizada em estudos sobre saúde, bem como sua versão em português.

5- Todas las veces que no me siento bien de salud, $1-2-3-4-5$ consulto un médico.

6 - Yo puedo controlar mi salud.

1-2-3-4-5

7- Si estoy enferma o con salud, mi familia tiene mucho que ver con eso.

8- Cuando enfermo, normalmente yo soy el culpable.

9- La suerte es muy importante para recuperarme de una enfermedad.

10- Quienes controlan mi salud, son los médicos.

$1-2-3-4-5$

11- Mi salud es principalmente una cuestión de suerte y azar.

12- La principal cosa que afecta mi salud, es lo que yo mismo hago.

13- Si yo me cuidara bien, puedo evitar enfermedades.

14- Cuando sano de una enfermedad, es porque las personas cuidaron bien de mí. (mi médico, mi esposa, la enfermera, los amigos, la familia, etc.)

15- No importa lo que yo haga, siempre es posible que esté enfermo.

16- Si fuera por mi destino, yo tendría salud.

17- Si yo hiciera las cosas bien, podría mantenerme

$1-2-3-4-5$

$1-2-3-4-5$

$1-2-3-4-5$

$1-2-3-4-5$

$1-2-3-4-5$

1-2-3-4-5

$1-2-3-4-5$ saludable.

18- Para tener salud, solo tengo que obedecer a mi médico.
$1-2-3-4-5$

1-2-3-4-5

1-2-3-4-5

$1-2-3-4-5$ 


\section{REFERÊNCIAS BIBLIOGRÁFICAS}

1. Rotter J. Generalized expectancies for internal versus external control of reinforcement. Psychol Monogr: Gen Appl 1966;80.

2. Dela Coleta JA. A escala de locus de controle interno-externo de Rotter: um estudo exploratório. Arq Bras Psicol 1979; 31 (4): 167-81. 3. Levenson $\mathrm{H}$. Activism and Powerful others: distinctions within the concept of internal-external control. J Pers Assess 1974; 38:377-3. 4. Wallston BS, Wallston KA, Kaplan GD, Maides SA. Development and validation of the Health Locus of Control (HLC) scale. J Consult Clin Psychol 1976; 44:580-5.

5. Wallston KA, Wallston BS, De Vellis R. Development of the multidimensional health locus of control (MHLC) Scales. Health Educ Monogr 1978; 6:161-70.

6. Furnham A, Steele H. Measuring locus of control: A critique of general, children's, health and work-related locus of control questionnaires. J Psychol 1983; 84:443-79.

7. Strickland BR. Internal-external expectancies and health-related behaviors. J Consult Clin Psychol 1978; 46(6):1192-211.

8. Wallston KA Wallston, BS. Locus of control and health: A review of the literature. Health Educ Monogr 1978;6:107-17.
9. Wallston KA, Wallston BS. Health locus of control scale. In: Lefcourt HM. Research with the Locus of Control Construct. New York: Academic Press; 1981. p.189-243.

10. Dela Coleta MF. Uso de métodos anticoncepcionais, aborto e motivação para a saúde. Resumos do Congresso Interamericano de Psicologia; 1991, julho 4-9. San Juan: SIP; 1991.

11. Dela Coleta MF. Prevenção da AIDS entre universitários sexualmente ativos. Resumos do 24 Congresso Interamericano de Psicologia. Santiago, Chile: SIP; 1993, maio 10-12.

12. Dela Coleta MF. O Modelo de Crenças em Saúde: uma aplicação a prevenção e controle da doença cardiovascular. [tese]. Brasília (DF): Universidade de Brasilia; 1995.

13. Dela Coleta JA. Atribuição de Causalidade: Teoria e Pesquisa. Rio de Janeiro: Fundação Getúlio Vargas; 1982.

14. Biaggio AMB. Relationships between strait-state anxiety and locus of control: experimental studies with adults and children. Munich (GE): International Society for the Study of Behavioral Development Biennial Meeting; 1983.

15. Dela Coleta MF. Escala Multidimensional de Locus de Controle de Levenson. Arq Bras Psicol 1987; 39 (2):79-97.

16. D'Amorin MAM. Internalidade, externalidade e explicações acerca do desemprego. Rev Psicol 1988; 6 (1):81-93. 